\title{
Design of a Wavelength Control for Coherent Detection of High Order Modulation Formats
}

\author{
Josep M. Fabrega* (1), Josep Prat* (1), L. Molle* (2), R. Freund (2), *Member, IEEE \\ (1) Universitat Politècnica de Catalunya; Jordi Girona 1-3; E-08034 Barcelona, Spain \\ e-mail:jmfabrega@tsc.upc.edu \\ (2) Fraunhofer Institute for Telecommunications, Heinrich-Hertz-Institut \\ Einsteinufer 37, 10587 Berlin, Germany,e-mail: freund@hhi.de
}

\begin{abstract}
An automatic frequency control design is proposed and demonstrated. It is based on a high-speed optical VCO. An overall design has been performed, based on simulation results. Also, loop delay and phase noise impacts have been evaluated. Frequency discriminator and VCO proposed have been experimentally characterized, showing an agreement with simulation results.
\end{abstract}

Keywords: frequency control, frequency estimation, coherent systems, phase modulation.

\section{INTRODUCTION}

Homodyne coherent optical reception received great attention at the beginning of the 90s. It presents many advantages with respect to the conventional direct detection because of its excellent wavelength selectivity, high sensitivity and tuneability performances. However, there have been never found practical application, mostly due to the complex receiver structure and the stringent linewidth and loop delay requirements [1]. Recently, some approaches to solve this have been proposed using ultra-fast digital signal processing to estimate and track the carrier phase [2-3]. However, all these techniques tolerate only a rather small frequency difference between LO and transmit laser. Thus, a very important point regarding receiver implementation is the frequency estimation and locking.

Until now, for the analog domain several designs have been proposed, coming from RF techniques [4], and being tested in optical communications [5]. Also, two main lines have been followed for the digital domain: the off-line processing and the real-time approach. For the off-line approach, the same receiver architecture is used, almost all times [6-7]. There the challenge is the development of feed-forward processing algorithms that can compensate the frequency offset between local laser and received signal.

In this paper we present, for the first time, a reliable, fast and real-time wavelength control for intradyne coherent reception, based on a single side-band optical VCO; intended for, at least, 8-PSK modulation format.

\section{LOOP DESIGN AND PERFORMANCES}

The overall loop proposed is shown in Fig. 1. It has a $90^{\circ}$ optical hybrid coherent reception front-end, driven by a local optical Voltage Controlled Oscillator (VCO); a delay-and-multiply frequency discriminator, and a loop control filter. The VCO device used is an optical VCO that can be controlled in a stable and fast way, like an electrical one. Its conceptual scheme is detailed on Fig. 1. The objective is to control a Single Side Band (SSB) tone modulation of the local laser. For that reason an IQ modulator is used. Details of its performance and characterization for using it in optical phase-locking can be found in [8].

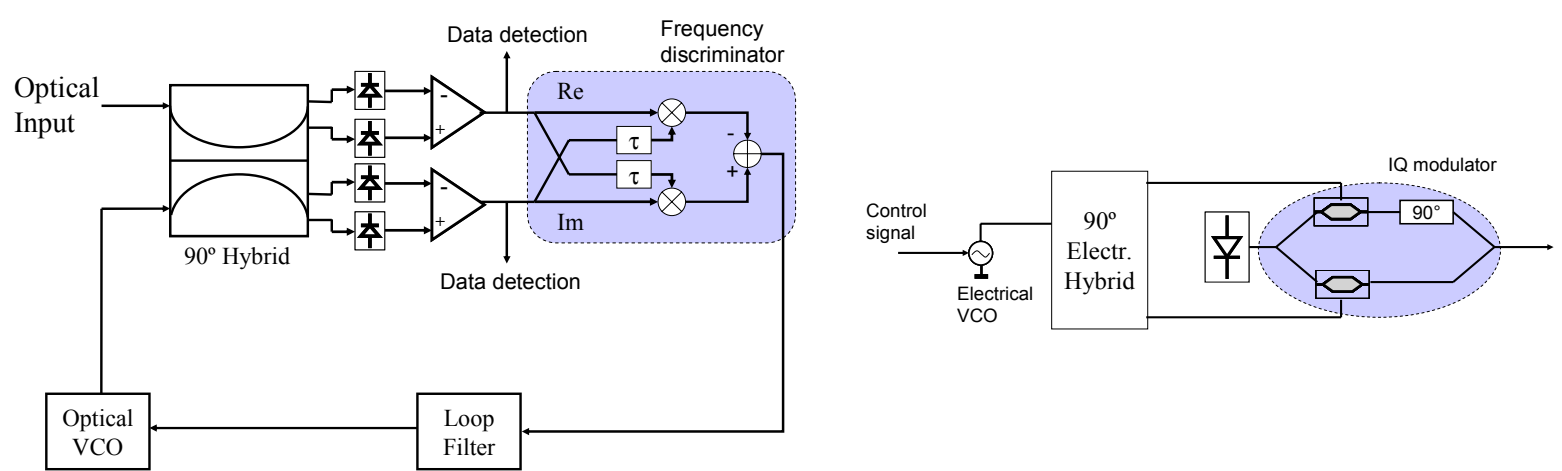

Fig. 1. Scheme of the proposed analog frequency estimation loop (left) and optical SSB-modulation VCO (right).

Regarding the frequency discriminator, we found that a good and reliable implementation can be just a delayand-multiply architecture that has been deeply studied and reported in [4, 9].

In order to evaluate the performances of the proposed receiver architecture, several sets of simulations were run for different parameters: frequency discriminator performances, loop delay impact and phase noise impact. 
Regarding frequency discriminator performances, we configured a first set of simulations of an RZ-8-PSK scheme, with a coherent receiver followed by a digitization stage. Since it is digitized, we could compare the proposed frequency discriminator with a theoretical solution that can be used in off-line processing: a linear fit of the phase curve. Results are shown in Fig. 2. There we can observe that, while the proposed scheme gets almost unaffected, the other has a discontinuity around $\pm 1250 \mathrm{MHz}$. This is because in the last case, a power-of-eight block is required to remove the 8-PSK modulation, and this puts a cycle-slipping indetermination on that frequency, that has to be taken into account when estimating the frequency.

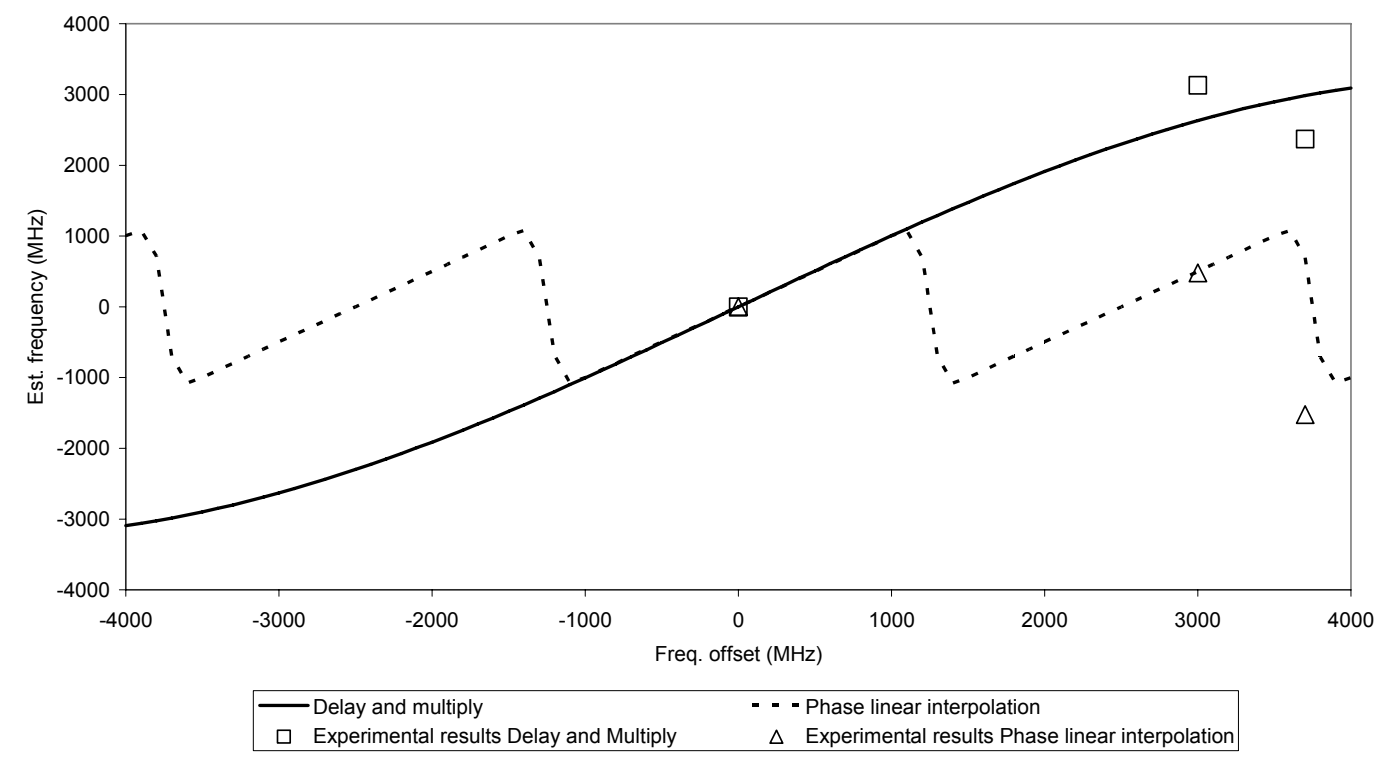

Fig. 2. Frequency discriminator output vs. frequency difference between $L O$ and received signal.

After characterizing the frequency discriminator with the proposed modulation, we made a numerical analysis departing from the theoretical equations that arise from loop linearization $[4,9]$. Regarding this numerical analysis, we found that the transfer function can be expressed as:

$$
H_{S}(S)=\frac{K \cdot K_{P} \cdot S+K \cdot K_{I}}{\left(1+K \cdot K_{P}\right) S+K \cdot K_{I}} ; \quad K=8 \pi K_{L O} \Re^{2} R_{L}^{2} P_{S} P_{L O} ; \quad T=\frac{1+K \cdot K_{P}}{K \cdot K_{I}}
$$

where $\mathrm{S}$ is the complex angular frequency, $K_{L O}$ is the sensitivity of the optical VCO module $(\mathrm{Hz} / \mathrm{V}), \Re$ is the photo-detector's responsivity, $R_{L}$ is the load impedance, $P_{S}$ is the received optical power, $P_{L O}$ is the local oscillator optical power, $K_{p}$ is the proportional gain of the loop filter, $K_{I}$ is the integral gain of the loop filter, and $T$ is the time constant of the system.

From this model, we evaluated the loop delay impact. The output parameter evaluated was the setting time at $10 \%$ error from the final value, for a low frequency step $(100 \mathrm{MHz})$. Results are shown in Fig. 3. There, we can see that when loop delay is about one fifth the time constant, the system performances decreases dramatically.

Afterwards we implemented a module for the full loop (no data), using the software VPItransmissionMaker. With this model, we evaluated the phase noise impact to the frequency control. Results are shown in Fig. 3. As we expected, it introduces a residual error, with Gaussian statistics, that cannot be tracked nor compensated at all. The variance of such error is proportional to the laser linewidth, as we could expect. Thus, this error can not be compensated at all, and a separate phase compensation method has to be used.
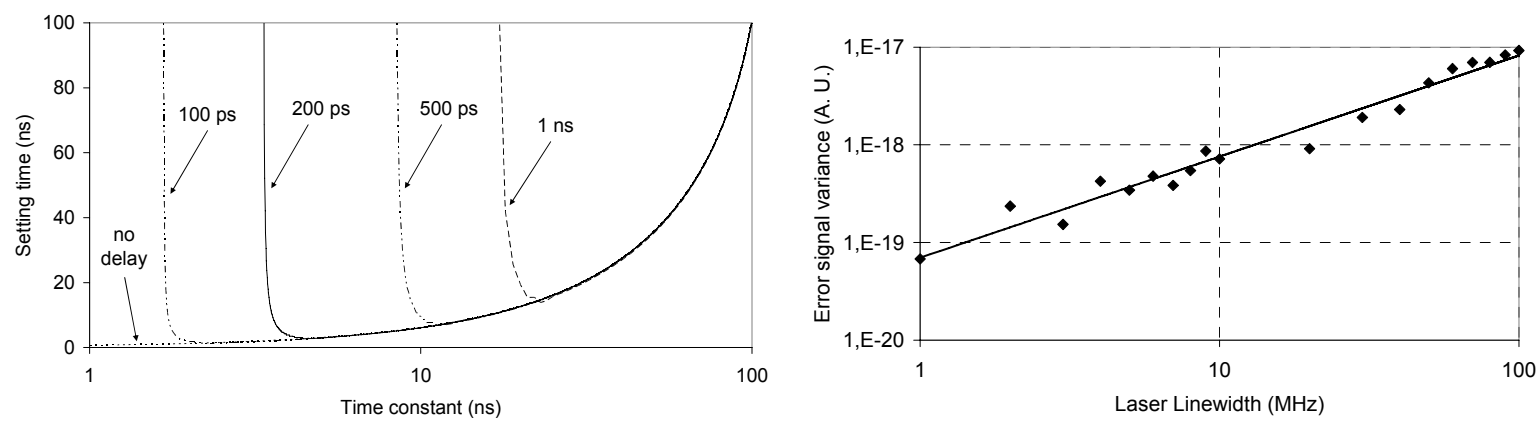

Fig. 3. Loop delay impact on loop setting time, for several loop delays (left). Error signal variance vs. laser linewidth (right). 


\section{PRACTICAL IMPLEMENTATION}

In Fig. 4 (left) a schematic of the overall loop to be implemented is shown. Of that loop we characterized the optical VCO and afterwards, with the SSB VCO, we have carried out some preliminary measurements (based on off-line processing) of the frequency estimator.

Max hold function for the output spectrum of the optical VCO
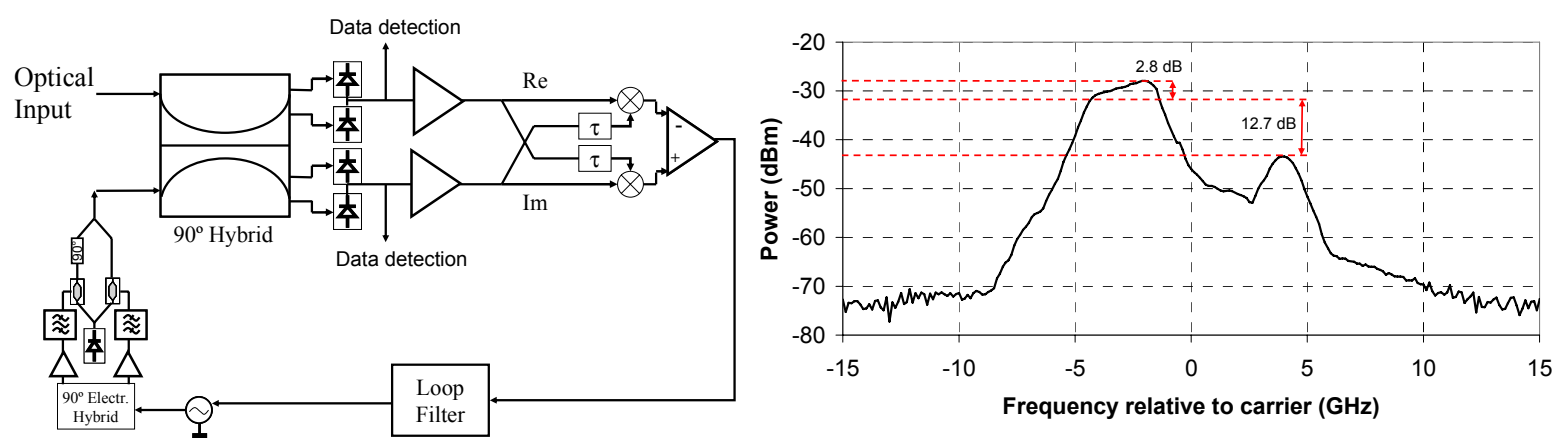

Fig. 4. Schematic to be implemented (left), Max hold function for the output spectrum of the optical VCO (right).

Regarding VCO characterization, a result of the maximum hold function for the output spectrum of the optical VCO is shown in Fig. 4 (right). There, we can see that such an optical VCO has a residual amplitude tilt, with a slope of $2.8 \mathrm{~dB}$. Also, when approaching to the limit of $\pm 4 \mathrm{GHz}$, a high peak appears at the opposite side of the spectrum. This peak corresponds to a worst case condition, and is $12.7 \mathrm{~dB}$ below the principal component. Precisely, this is shown in Fig. 4 (right) for the $-4 \mathrm{GHz}$ case. That range of $4 \mathrm{GHz}$ is set by the RF components used (hybrid, amps and filters) and is enough for our purposes. Of course, by carefully setting these RF components, the performances of the VCO can be substantially improved.

Afterwards, we compared it to other two optical VCO approaches. Results are shown in table 1. One approach is based on tuning the current of the phase section of a GCSR laser. It presents some difficulties for setting the appropriate working point, and could present some hysteresis, depending on the wavelength range we are operating [10]. Data presented here has been obtained from [10], where a GCSR laser model NYW 30-009 from ALTITUN, was characterized. A second alternative VCO is a DFB laser, working in a saturation point and modulating its adiabatic chirp for changing the wavelength. However it could give a high residual amplitude change. For this approach, we retrieved data obtained from basic measurements carried out of a Panasonic LNFE03YBE1UP.

Table 1. Comparison between possible optical VCO approaches.

\begin{tabular}{|l|c|c|c|c|c|}
\hline & Linewidth & Tuning speed & Tuning range & Freq. slope & Residual Amp. change \\
\hline SSB-IQ mod. & $100 \mathrm{kHz}$ & $10 \mathrm{MHz}$ & $2 \mathrm{GHz}$ & $260 \mathrm{MHz} / \mathrm{V}$ & $2.8 \mathrm{~dB}$ \\
\hline GCSR & $60 \mathrm{MHz}$ & $100 \mathrm{MHz}$ & $18 \mathrm{GHz}$ & $320 \mathrm{MHz} / \mathrm{V}$ & $0.1 \mathrm{~dB}$ \\
\hline DFB & $1 \mathrm{MHz}$ & $1 \mathrm{GHz}$ & - & $1.4 \mathrm{GHz} / \mathrm{V}$ & $7.4 \mathrm{~dB}$ \\
\hline
\end{tabular}

In order to see the potential when using the proposed frequency discriminator, we carried out some off-line experiments. The setup used is depicted in Fig. 5. It is a back-to-back version of the 10 GBaud RZ-8PSK (30 Gbps) transmission system reported in [11], with the proposed optical VCO. There, the transmitter consists of a laser that for RZ pulse carving a Mach-Zehnder Modulator (MZM) is used. Regarding the 8-PSK signal, it was generated by an IQ-modulator, which generated an optical QPSK signal, and a consecutive phase modulator, which was used for the additional $\pi / 4$ phase modulation. The transmitted data signal was a $2^{11}-1$ pseudo-random bit sequence, given by a Pulse-Pattern Generator (PPG). These signals were given to the modulator inputs with different delays to ensure their independence.

At the receiver side, the RZ-8-PSK signal was interfered with the optical VCO in a $\mathrm{LiNbO}_{3} 2 \times 490^{\circ}$ hybrid. The output signals of the hybrid were detected by two balanced detectors, and the I and Q outputs were sampled using a $50 \mathrm{GSa} / \mathrm{s}$ digital storage oscilloscope.

In Fig. 2, we can see the estimation results, for two different discriminators: Linear curve fitting, and delay and multiply method. We compared between 3 different cases: No frequency difference, $3 \mathrm{GHz}$ of frequency difference, and $3.5 \mathrm{GHz}$ of frequency difference. There, we can see that for all cases of frequency difference, both methods, linear fitting and delay-and-multiply, work as expected, but with a non-negligible error. Those differences are attributable to the laser instability. 


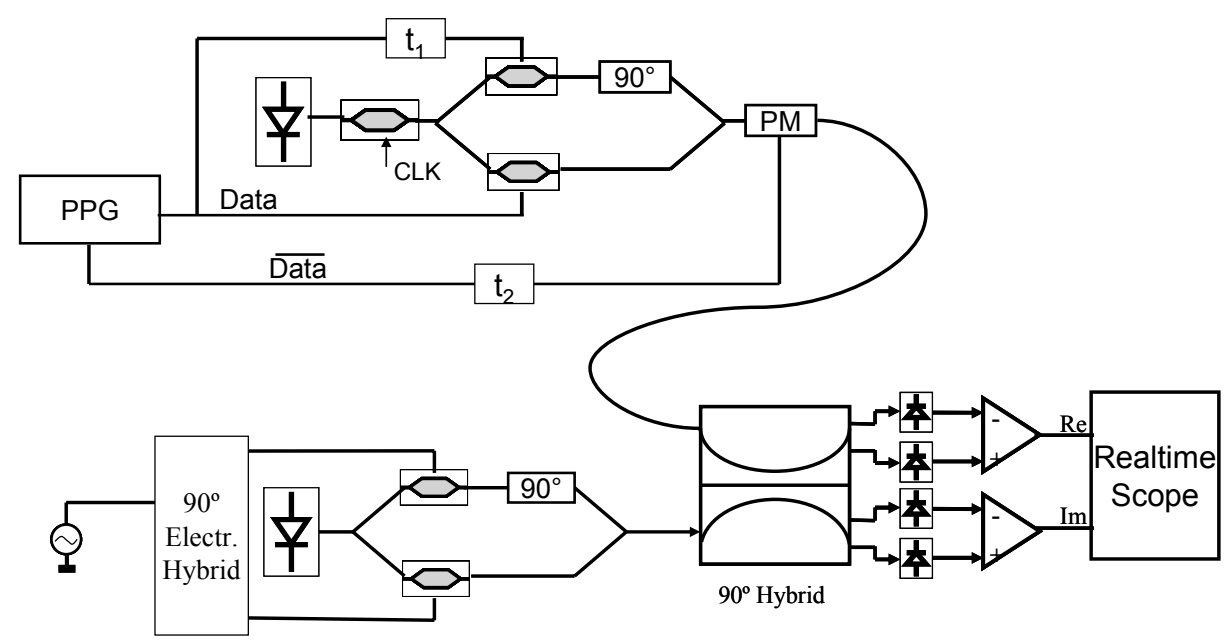

Fig. 5. Experimental setup (left), Frequency estimation vs. frequency drift (right).

\section{CONCLUSIONS}

An automatic frequency control design has been proposed and demonstrated. It is based on a high-speed optical VCO. First proof-of-concept experiments have been performed, showing the feasibility of the main components of the loop and a good agreement with simulation results.

\section{ACKNOWLEDGEMENTS}

The work reported in this paper was supported in part by the FP7 BONE project, the FP7 EUROFOS project, and the Spanish MEC PTA-2003-02-00874 grant.

\section{REFERENCES}

[1] L. Kazovsky, "Homodyne phase-shift-keying systems: Past challenges and future opportunities," in Proc. OFC 2005, Anaheim, OTuL3 (2005).

[2] M. G. Taylor, "Coherent detection method using DSP for demodulation of signal and subsequent equalization of propagation impairments," Photonics Technology Letters 16, 674-676 (2004).

[3] R. Noé, "Phase noise-tolerant synchronous QPSK/BPSK baseband-type intradyne receiver concept with feedforward carrier recovery,", Journal of Lightwave Technology 23, 802-808 (2005).

[4] F. D. Natali, "AFC Tracking Algorithms," IEEE Trans. on Communications, vol. 32, no. 8, Aug. 1984.

[5] R. Noe, W. B. Sessa, R. Welter, L. G. Kazovsky, "New FSK Phase-Diversity Receiver in a $150 \mathrm{Mbit} / \mathrm{s}$ Coherent Optical Transmission System," IET Electronics Letters, vol. 24, no. 9, Apr. 1988.

[6] L. Li, Z. Tao, S. Oda, T. Hoshida, J. C. Rasmussen, "Wide-range, accurate and simple digital frequency offset compensator for optical coherent receivers," in Proc. OFC08, paper OWT4.

[7] A. Leven, N. Kaneda, K. Ut-Va Koc, Y-K. Chen, "Frequency estimation in intradyne reception," IEEE Photonics Technology Letters, vol. 19, no. 6, Mar. 2007.

[8] V. Ferrero, S. Camatel, "Optical phase locking techniques: An overview and a novel method based on single side sub-carrier modulation," OSA Optics Express, vol. 16, no. 2, Jan. 2008.

[9] H. Meyr, G. Acheid, "Synchronization in Digital Communications vol. 1," 1990, John Wiley \& Sons.

[10] V. Polo, "Contribution to the dynamic tuning of tunable laser sources for modulation and optical packet routing in WDM and access networks," Universitat Politècnica de Catalunya, Ph. D. Thesis, 2006.

[11] R. Freund, D.-D. Gross, M. Seimetz, L. Molle, C. Caspar, "30 Gbit/s RZ-8-PSK transmission over 2800 $\mathrm{km}$ standard single mode fibre without inline dispersion compensation," in Proc. OFC08, paper OMI8. 